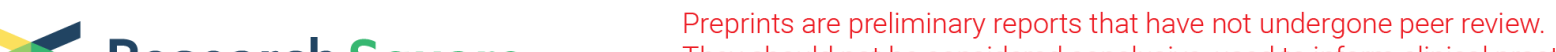 Research Square They should not be considered conclusive, used to inform clinical practice, or referenced by the media as validated information.
}

\section{Biological-guided Metronomic Chemotherapy as Maintenance Strategy in Responders After Induction Therapy in Metastatic Colorectal Cancer}

\section{Min Shi}

Shanghai Jiao Tong University Medical School Affiliated Ruijin Hospital

Tao Ma

Shanghai Jiao Tong University Medical School Affiliated Ruijin Hospital

\section{Wenqi Xi}

Shanghai Jiao Tong University Medical School Affiliated Ruijin Hospital

Jingling Jiang

Shanghai Jiao Tong University Medical School Affiliated Ruijin Hospital

Junwei Wu

Shanghai Jiao Tong University Medical School Affiliated Ruijin Hospital

Chenfei Zhou

Shanghai Jiao Tong University Medical School Affiliated Ruijin Hospital

Chen Yang

Shanghai Jiao Tong University Medical School Affiliated Ruijin Hospital

Zhenggang Zhu

Shanghai Jiao Tong University Medical School Affiliated Ruijin Hospital Jun Zhang ( $\nabla$ junzhang10977@sjtu.edu.cn )

Shanghai Jiao Tong University Medical School Affiliated Ruijin Hospital

\section{Study protocol}

Keywords: metronomic chemotherapy, capecitabine, maintenance treatment, metastatic colorectal cancer

Posted Date: November 13th, 2019

DOI: https://doi.org/10.21203/rs.2.17153/v1

License: (c) (i) This work is licensed under a Creative Commons Attribution 4.0 International License.

Read Full License 


\section{Abstract}

Background The aim of this study is to demonstrate that capecitabine metronomic chemotherapy is noninferior to capecitabine conventional chemotherapy as maintenance treatment, who have responded to 16-18 weeks first-line chemotherapy in metastatic colorectal cancer.

Methods The study design is a prospective, randomized, open label, phase II clinical trial. Those mCRC patients who respond well after 16-18 weeks of standard doublet chemotherapy as induction may enrolled into this study, randomly divided into capecitabine metronomic group or standard dosage group. The duration of disease control after randomization and progression free survival from enrollment are primary endpoints. Meanwhile, the overall survival, safety and quality of life are secondary endpoints. The sample size required to achieve the research objectives of this project is 79 cases in each group. The study recently started on 29-01-2018, and will last for 36 months.

Discussion This project intends to study the efficacy and safety of capecitabine metronomic chemotherapy in the maintenance treatment of advanced colorectal cancer, and to explore the strategy of "low toxicity, high efficiency, economy and individualization" which is suitable for China's national conditions and pharmacoeconomics. It has great clinical application prospects and clear socio-economic value.

\section{Background}

Global Cancer Statistics 2018 indicated that there would be an estimated 18.1 million newly diagnosed cancer cases and 9.6 million cancer-related deaths in 2018. Among them, over 1.8 million new colorectal cancer cases and 881000 deaths were estimated to occur in 2018. Overall, colorectal cancer ranked third in all cancer incidence (6.1\%) and second for mortality (9.2\%). The incidence rates of colorectal cancer are about 3-fold higher in transitioned versus transitioning countries[1]. The difference may due to dietary patterns, obesity, and lifestyle factors. Standard screening and early detection programs have been conducted in the United States and Japan since 1990s[2], and the 5-year survival rate of colorectal cancer was increased from $51 \%$ to $65 \%$ while more and more patients were diagnosed as early staging. Even so, there will be almost half of the colorectal cancer patients eventually develop metastasis and lose the chance to eradicate cancer[3]. For these patients, how to prolong the survival time and inhibit the growth of tumors on the premise of guaranteeing the quality of life, and transform the metastatic colorectal cancer ( $\mathrm{mCRC}$ ) into chronic diseases like diabetes and hypertension through long-term, low toxicity and effective drug treatment are of great clinical research value.

Drug therapies for mCRC patients were ranged from 5-fluorouracil monotherapy in the 1960s to 5fluorouracil in combination with oxaliplatin or irinotecan and with or without targeted agents such as bevacizumab, cetuximab or panitumumab in the past decade. The median overall survival (OS) of mCRC patients was from less than 12 months to more than 33 months[4-10]. However, conventional chemotherapy usually gives the maximum tolerable dose of the drug, and will cause huge toxicity and 
side effects while killing cancer cells. Chemotherapy-related vomiting, diarrhea, agranulocytosis, peripheral neurotoxicity and other serious adverse reactions occur as high as $5-20 \%[6,11-13]$. It takes a period of time for the body to recover from toxic and side effects after each routine chemotherapy administration, and repeated multiple cycles of administration are more likely to cause toxicity accumulation, which limits the number of courses of treatment. More importantly, after a period of highintensity chemotherapy, how to continue to effectively and persistently inhibit the progress of cancer, while ensuring patients with good tolerance and quality of life, has been a hot topic in cancer research, but also a clinical problem to be solved urgently.

Metronomic chemotherapy is a low-dose, high-frequency mode of continuous administration of antineoplastic drugs without long intermission[14]. The recommended dose is only $1 / 10-1 / 3$ of the maximum tolerable dose of the drug, so the incidence and intensity of treatment-related side effects are greatly reduced. The antineoplastic mechanism of metronomic chemotherapy is not directed against cancer cells, therefore, it will not produce the problem of drug resistance induced by small doses of drugs. By inhibiting the proliferation and migration of vascular endothelial cells, metronomic chemotherapy is also known as "anti-angiogenesis chemotherapy"[15].

\section{Methods}

\section{Aim of the study}

The aim of this study is to demonstrate that capecitabine metronomic chemotherapy is non-inferior to capecitabine conventional chemotherapy as maintenance treatment, who have responded to 16-18 weeks first-line chemotherapy in $\mathrm{mCRC}$.

\section{Study design}

The study design is a prospective, randomized, open label, phase II clinical trial (Figure 1). Those mCRC patients who respond well, stable disease (SD), partial response (PR) or complete response (CR) according to RECIST Criteria after 16-18 weeks of standard doublet chemotherapy as induction may enrolled into this study, randomly divided into capecitabine metronomic group or standard dosage group. Randomization was done by sealed envelope system. The maintenance treatments are continued until disease progression or severe toxicity. Furthermore, exploratory markers involving angiogenesis (serum VEGF, PDGF, Tie-1 and Tie2, etc) and immune function (CD clusters, serum tumor mutation burden (TMB), etc), are conducted via liquid biopsy (Figure 2).

\section{Study objectives}

The duration of disease control after randomization (progression free survival 2, PFS2) and progression free survival from enrollment (PFS1) are primary endpoints. Meanwhile, the OS, safety and quality of life 
(QoL) are secondary endpoints.

\section{Study population}

The study population consists of patients with unresectable metastatic colorectal cancer, who are scheduled for treatment with first-line doublet chemotherapy. Patients' inclusion and exclusion criteria are defined as follows:

Inclusion criteria:

- Patients of an age from 18 to 75 years;

- Histopathologically confirmed colorectal adenocarcinoma and classified as technically unresectable (patients with only local recurrence are not eligible);

- No prior first line treatment of chemotherapy, radiotherapy, immunotherapy or targeted therapy; Adjuvant chemotherapy is allowed if it has been more than 6 months since the treatment was finished and there have been no signs of disease progression, neither during treatment nor during the 6 months following its completion.

- Life expectancy > 12 weeks;

- Eastern Cooperative Oncology Group (ECOG) performance status $\leq 1$;

- At least one measurable lesion for assessment by computed tomography (CT) or magnetic resonance imaging (MRI);

- Adequate bone marrow function $(\mathrm{Hb}>6.0 \mathrm{mmol} / \mathrm{L}$, absolute neutrophil count $>1.5 \times 109 / \mathrm{L}$, platelets $>100 \times 109 / L$ ), renal function (serum creatinine $\leq 1.5 \times$ ULN and creatinine clearance, Cockroft formula, $>30 \mathrm{ml} / \mathrm{min}$ ), liver function (serum bilirubin $\leq 2 \times$ ULN, serum transaminases $\leq 3 \times$ ULN without presence of liver metastases or $\leq 5 x$ ULN with presence of liver metastases);

- Disease evaluation with proven SD, PR or CR according to RECIST after first-line induction treatment before randomization;

- Written informed consent should be obtained before randomization;

Exclusion criteria:

- Brain metastasis and with large amounts of pleural and abdominal effusion;

- Pregnancy or breastfeeding;

- Disease evaluation with Progression disease (PD) according to RECIST after first-line induction treatment;

- Previous systemic treatment for advanced disease;

- Major surgery or radiotherapy (except for antalgic surgery that does not include measurable target lesions) during the 4 weeks prior to inclusion in the study;

- Participation in another clinical trial with drugs within the previous 30 days; 
- Neoplasm in the 2 years prior to entering the study, except for non-melanoma skin carcinoma or in situ cervix carcinoma;

- With symptomatic heart disease (arrhythmia, heart failure, or history of myocardial infarction);

- With active infection, active bleeding or serious metabolic disorder;

- Signs and symptoms, at the moment of entering the study, of acute or subacute bowel obstruction;

- Chronic immunological or hormonal treatment, except for hormone replacement treatment at physiological doses.

- Any geographical or social circumstance or any medical or psychological alteration that, in the investigator's opinion, will not allow the patient to conclude the study.

\section{Study protocol}

\section{MDT}

Ideally, patients will be discussed by the multi-disciplinary team (MDT) for colorectal cancer from Departments of Surgery, Oncology, Radiology, Pathology, Nutrition and Interventional medicine, etc.) of Ruijin Hospital, Shanghai Jiaotong University School of Medicine, and first-line chemotherapy regimen was formulated by the joint consultation of these experts.

\section{First-line treatment regimens}

Standard doublet chemotherapies were used as induction treatment which including mFOLFOX6 regimen (oxaliplatin $85 \mathrm{mg} / \mathrm{m} 2$ iv d1, leucovorin $400 \mathrm{mg} / \mathrm{m} 2$ iv d1, 5-fluorouracil $400 \mathrm{mg} / \mathrm{m} 2$ iv d1, 5-fluorouracil $2400 \mathrm{mg} / \mathrm{m} 2$ CIV 46h, q2w), FOLFIRI regimen (irinotecan $180 \mathrm{mg} / \mathrm{m} 2$ iv d1, leucovorin $400 \mathrm{mg} / \mathrm{m} 2$ iv d1, 5fluorouracil $400 \mathrm{mg} / \mathrm{m} 2$ iv d1, 5-fluorouracil $2400 \mathrm{mg} / \mathrm{m} 2$ CIV $44 \mathrm{~h}, \mathrm{q} 2 \mathrm{w}$ ), XELOX regimen (oxaliplatin $135 \mathrm{mg} / \mathrm{m} 2$ iv $\mathrm{d} 1$, capecitabine $1000 \mathrm{mg} / \mathrm{m} 2$ bid po d1 $1-14$, q3w), XELIRI regimen (irinotecan $250 \mathrm{mg} / \mathrm{m} 2$ iv $\mathrm{d} 1$, capecitabine $1000 \mathrm{mg} / \mathrm{m} 2$ bid po $\mathrm{d} 1-14, \mathrm{q} 3 \mathrm{w}$ ). Total first-line treatments were 6 cycles for XELOX/XELIRI regimens, and 8 cycles for mFOLFOX6/FOLFIRI regimens.

\section{Maintenance treatment regimens}

Single-agent chemotherapy was used as maintenance treatment which including capecitabine metronomic chemotherapy (capecitabine $500 \mathrm{mg}$ bid po), capecitabine conventional chemotherapy (capecitabine $1000 \mathrm{mg} / \mathrm{m} 2$ bid po, d1-14, q3w).

\section{Outcome measurements}


Evaluation of tumor response was performed every 8 weeks by the response evaluation in solid tumors criteria (RECIST)[16]. Toxicity was assessed after each cycle by using National Cancer Institute Common Toxicity Criteria for Adverse Events (NCl-CTCAE)[17]. Quality of life was assessed after each cycle by the European Organization for Research and Treatment of Cancer Quality of Life Questionnaire.

\section{Sample size calculation}

This project is a non-inferior study. The capecitabine metronomic chemotherapy group (experimental group) and capecitabine conventional chemotherapy group (control group) were randomly divided into two groups with a sample size of $1: 1$. When the lower limit of $95 \%$ confidence interval is not less than 0.9 ( $a=0.05, \beta=0.2$, power: $90 \%, \delta=0.15$ ), the clinical efficacy of metronomic chemotherapy group can be judged as not inferior to that of conventional chemotherapy. Using NCSS-PASS 11 software to estimate the required sample size, the sample size required to achieve the research objectives of this project is 79 cases in each group. Considering the loss of patients (less than 20\%) and the assessment of disease progression after first-line treatment (about 20\%), randomized grouping sample size was 200 cases and initial screening sample size was 250 cases.

\section{Statistical analysis}

The statistical analysis was carried out using SPSS software (version 17.0; SPSS, Chicago, IL, USA). Descriptive statistics were used for safety evaluation. Mean values and standard deviations (SDs) were provided for continuous endpoints and frequency and percentage distributions were provided for discrete data. PFS and OS were estimated using the Kaplan-Meier method and their medians along with two-sided 95\% Cis were calculated. Comparisons between groups of patients were made by the log-rank test. All statistical analysis were carried out at a $5 \%$ level significance.

\section{Discussion}

Until recently, few mCRC patients could tolerate full doses of chemotherapy longer than 4-6 months, The limitations are mainly due to severe neurotoxicity (oxaliplatin) and chronic diarrhea (irinotecan)[18]. Thus, limiting the duration of the induction chemotherapy to a short period, then exploiting maintenance to prolong disease control at the price of a reasonable toxicity profile, is an appealing strategy for $\mathrm{mCRC}$ patients[19].

Lots of studies aimed to reduce the treatment burden and maintain a favorable outcome. OPTIMOX1 trial compared 5FU/LV maintenance treatment with continuous FOLFOX4 regimen in mCRC patients and found that there were no significance in PFS, OS and incidence of adverse events between the two groups, which suggesting that fluorouracil could be used as an alternative maintenance therapy during the standard regimen treatment without affecting the overall therapeutic effect[20]. MACRO trial compared the efficacy and safety of bevacizumab alone with bevacizumab plus capecitabine and 
oxaliplatin as maintenance treatment after induction chemotherapy in MCRC patients, which suggests that single-agent bevacizumab as maintenance therapy may be an appropriate option following induction XELOX plus bevacizumab in mCRC patients with mild improvement in PFS[21]. MACRO 2 trial compared the efficacy and safety of cetuximab alone with cetuximab plus mFOLFOX as maintenance treatment after induction chemotherapy in MCRC patients, there were no statistically significant differences in the PFS and OS, the objective response rate and safety profile were also similar. Which suggests that maintenance therapy with single-agent cetuximab following mFOLFOX+cetuximab induction could be a valuable option compared with mFOLFOX+cetuximab treatment continuation[22]. CAIRO3 study reported metronomic capecitabine combined with bevacizumab as maintenance treatment in mCRC patients, it concluded that the PFS of capecitabine metronomic chemotherapy combined with bevacizumab maintenance group was significantly longer than that of the observation group, and the incidence of chemotherapy-related leukopenia, peripheral neurotoxicity and other serious toxic reactions was only increased by $5-10 \%$ compared with the observation group, which was completely tolerated by the patients, which has been proved to be an effective and low toxicity maintenance therapy strategy[23]. But bevacizumab is expensive, cost-effectiveness of maintenance capecitabine and bevacizumab for $\mathrm{mCRC}$ has been calculated and found that antineoplastic therapy is expensive for payers and society. The price of capecitabine and bevacizumab maintenance therapy should need to be reduced by $93 \%$ to make it cost-effective, which restricted the clinical application of this combination regimen as maintenance treatment in $\mathrm{MCRC}$ patients.

$\mathrm{Xu}$ et al. [24]reported the results of single-agent capecitabine as maintenance therapy after induction firstline chemotherapy of $\mathrm{mCRC}$, the primary endpoint of PFS in capecitabine maintenance group (capecitabine $1000 \mathrm{mg} / \mathrm{m} 2$ bid po, d1-14, q3w) was 6.43 months ( $95 \% \mathrm{Cl} 5.26-7.71$ ), which was significantly longer than the observation group (3.43 months, $95 \% \mathrm{Cl} 2.83-4.16), \mathrm{HR}=0.54(0.42-0.70)$, $\mathrm{P}<0.001$. However, the maximum tolerable dose of capecitabine for maintenance therapy was not adjusted, resulting in the incidence of grade $3 / 4$ toxicity in maintenance treatment group as high as $41 \%$, especially in leukopenia, thrombocytopenia, hand-foot syndrome and mucositis, which was significantly higher than that in observation group.

In recent years, metronomic chemotherapy, as a maintenance therapy strategy for advanced tumors, has been more and more used in clinic and become a new hotspot of anti-cancer therapy. It is a promising strategy for inhibiting angiogenesis and is associated with lower toxicities than conventional chemotherapy[25]. Our previous publication reported that capecitabine metronomic chemotherapy could decrease vascular endothelial growth factor (VEGF) while elevate thrombospondin-1 (TSP-1) expression, an endogenous inhibitor of angiogenesis. And then it could reduce CEP levels and decrease microvessel density (MVD)[26]. Our findings indicated that target angiogenesis rather than drug-sensitive tumor cells was the antitumor effects of capecitabine metronomic treatment in colon cancer cells. With regard to immunomodulation and tumor microenvironments, there were publications reported that metronomic chemotherapy could restore peripheral T-cell proliferation and activate the cytotoxicity of immune effector cells, which could inhibit tumor progression[27]. Besides this, metronomic chemotherapy could also upregulate dendritic cells to stimulated the proliferation of T lymphocytes[28]. Interestingly, it 
has been reported that metronomic chemotherapy could result in tumor immunogenicity with antigen processing and presentation genes.

The concepts were emphasized in the design of the BIG-MOON study, where a shortened induction phase, limited to 8 instead of 12 cycles (mFOLFOX6/FOLFIRI regimens), 6 instead of 8 cycles (XELOX/XELIRI regimens), followed by two different maintenance strategies. Besides, exploratory markers involving angiogenesis (serum VEGF, PDGF, Tie-1 and Tie2, etc) and immune function (CD clusters, serum tumor mutation burden (TMB), etc) are conducted via liquid biopsy.

In conclusion, the BIG-MOON study is a prospective study evaluating whether the effect of capecitabine metronomic chemotherapy as maintenance treatment is non-inferior to capecitabine conventional chemotherapy, who have responded to 16-18 weeks first-line chemotherapy in mCRC. This project intends to study the efficacy and safety of capecitabine metronomic chemotherapy in the maintenance treatment of advanced colorectal cancer, and to explore the strategy of "low toxicity, high efficiency, economy and individualization" which is suitable for China's national conditions and pharmacoeconomics. It has great clinical application prospects and clear socio-economic value.

\section{Declarations}

\section{Trial status}

This is protocol version 20170407. Enrollment started on 29-01-2018, and will last for 36 months. After the start of the study, the first 30 months will consist of inclusion and follow-up of the patients. The last 6 months will consist of follow-up and analysis of results. The study will end at 29-01-2021.

\section{Abbreviations}

mCRC, metastatic colorectal cancer; OS, overall survival; SD, stable disease; PR, partial response; CR, complete response; TMB, tumor mutation burden; PFS, progression free survival; QoL, quality of life; ECOG, Eastern Cooperative Oncology Group; CT, computed tomography; MRI, magnetic resonance imaging; PD, Progression disease; MDT, multi-disciplinary team; RECIST, response evaluation in solid tumors criteria; NCI-CTCAE, National Cancer Institute Common Toxicity Criteria for Adverse Events; SDs, standard deviations; VEGF, vascular endothelial growth factor; TSP-1, thrombospondin-1; MVD, microvessel density.

\section{Ethics approval and consent to participate}

This trial was approved by the Ethics committee of Ruijin Hospital affiliated to Shanghai Jiaotong University School of Medicine. Written informed consents were obtained from all participants prior to enrollment according to the Declaration of Helsinki. 


\section{Consent for publication}

Not applicable

\section{Availability of data and materials}

All data generated or analyzed during this study are included in this paper.

\section{Competing interests}

The authors declare that they have no competing interests.

\section{Funding}

The study was supported by National Science Foundation of China (81672327) and Program of Shanghai Academic/Technology Research Leader (17XD1402600) and Program for Outstanding Medical Academic Leader and Shanghai Municipal Education Commission-Gaofeng Clinical Medicine Grant Support (20161410) and Development Grant for Clinical Trial (SHDC12017X06). This is an investigator initiated study. There is no role for the funding body in collecting, analyzing and interpreting data and in writing the manuscript.

\section{Authors' contributions}

This study was conceived and designed by JZ, ZZ, MS. MS, TM, WX, JJ, JW, CZ, CY were responsible for clinical input. MS, JZ drafted the paper. All authors provided significant input to the paper by means of revisions and have read and approved the final manuscript.

\section{Acknowledgements}

None.

\section{References}

1.Bray F, Ferlay J, Soerjomataram I, Siegel RL, Torre LA, Jemal A. Global cancer statistics 2018:

GLOBOCAN estimates of incidence and mortality worldwide for 36 cancers in 185 countries. CA Cancer J Clin. 2018.

2.Schreuders EH, Ruco A, Rabeneck L, Schoen RE, Sung JJ, Young GP, et al. Colorectal cancer screening: a global overview of existing programmes. Gut. 2015; 64: 1637-49. 
3.Siegel R, Ma J, Zou Z, Jemal A. Cancer statistics, 2014. CA Cancer J Clin. 2014; 64: 9-29.

4.Heinemann V, von Weikersthal LF, Decker T, Kiani A, Vehling-Kaiser U, Al-Batran SE, et al. FOLFIRI plus cetuximab versus FOLFIRI plus bevacizumab as first-line treatment for patients with metastatic colorectal cancer (FIRE-3): a randomised, open-label, phase 3 trial. Lancet Oncol. 2014; 15: 1065-75.

5.Goldberg RM, Sargent DJ, Morton RF, Fuchs CS, Ramanathan RK, Williamson SK, et al. A randomized controlled trial of fluorouracil plus leucovorin, irinotecan, and oxaliplatin combinations in patients with previously untreated metastatic colorectal cancer. J Clin Oncol. 2004; 22: 23-30.

6.de Gramont A, Figer A, Seymour M, Homerin M, Hmissi A, Cassidy J, et al. Leucovorin and fluorouracil with or without oxaliplatin as first-line treatment in advanced colorectal cancer. J Clin Oncol. 2000; 18: 2938-47.

7.Hoff PM, Ansari R, Batist G, Cox J, Kocha W, Kuperminc M, et al. Comparison of oral capecitabine versus intravenous fluorouracil plus leucovorin as first-line treatment in 605 patients with metastatic colorectal cancer: results of a randomized phase III study. J Clin Oncol. 2001; 19: 2282-92.

8.Van Cutsem E, Kohne CH, Hitre E, Zaluski J, Chang Chien CR, Makhson A, et al. Cetuximab and chemotherapy as initial treatment for metastatic colorectal cancer. N Engl J Med. 2009; 360: 1408-17.

9. Hurwitz H, Fehrenbacher L, Novotny W, Cartwright T, Hainsworth J, Heim W, et al. Bevacizumab plus irinotecan, fluorouracil, and leucovorin for metastatic colorectal cancer. N Engl J Med. 2004; 350: 233542.

10.Douillard JY, Siena S, Cassidy J, Tabernero J, Burkes R, Barugel M, et al. Final results from PRIME: randomized phase III study of panitumumab with FOLFOX4 for first-line treatment of metastatic colorectal cancer. Ann Oncol. 2014; 25: 1346-55.

11.Douillard JY, Cunningham D, Roth AD, Navarro M, James RD, Karasek P, et al. Irinotecan combined with fluorouracil compared with fluorouracil alone as first-line treatment for metastatic colorectal cancer: a multicentre randomised trial. Lancet. 2000; 355: 1041-7.

12.Bokemeyer C, Bondarenko I, Hartmann JT, de Braud F, Schuch G, Zubel A, et al. Efficacy according to biomarker status of cetuximab plus FOLFOX-4 as first-line treatment for metastatic colorectal cancer: the OPUS study. Ann Oncol. 2011; 22: 1535-46.

13.Van Cutsem E, Kohne CH, Lang I, Folprecht G, Nowacki MP, Cascinu S, et al. Cetuximab plus irinotecan, fluorouracil, and leucovorin as first-line treatment for metastatic colorectal cancer: updated analysis of overall survival according to tumor KRAS and BRAF mutation status. J Clin Oncol. 2011; 29: 2011-9.

14.Weitman SD, Glatstein E, Kamen BA. Back to the basics: the importance of concentration $x$ time in oncology. J Clin Oncol. 1993; 11: 820-1. 
15.Kareva I, Waxman DJ, Lakka Klement G. Metronomic chemotherapy: an attractive alternative to maximum tolerated dose therapy that can activate anti-tumor immunity and minimize therapeutic resistance. Cancer Lett. 2015; 358: 100-6.

16.Therasse P, Arbuck SG, Eisenhauer EA, Wanders J, Kaplan RS, Rubinstein L, et al. New guidelines to evaluate the response to treatment in solid tumors. European Organization for Research and Treatment of Cancer, National Cancer Institute of the United States, National Cancer Institute of Canada. J Natl Cancer Inst. 2000; 92: 205-16.

17.Trotti A, Colevas AD, Setser A, Rusch V, Jaques D, Budach V, et al. CTCAE v3.0: development of a comprehensive grading system for the adverse effects of cancer treatment. Semin Radiat Oncol. 2003; 13: $176-81$.

18.Berry SR, Cosby R, Asmis T, Chan K, Hammad N, Krzyzanowska MK. Continuous versus intermittent chemotherapy strategies in metastatic colorectal cancer: a systematic review and meta-analysis. Ann Oncol. 2015; 26: 477-85.

19.Cremolini C, Marmorino F, Bergamo F, Aprile G, Salvatore L, Masi G, et al. Phase II randomised study of maintenance treatment with bevacizumab or bevacizumab plus metronomic chemotherapy after first-line induction with FOLFOXIRI plus Bevacizumab for metastatic colorectal cancer patients: the MOMA trial. Eur J Cancer. 2019; 109: 175-82.

20.Tournigand C, Cervantes A, Figer A, Lledo G, Flesch M, Buyse M, et al. OPTIMOX1: a randomized study of FOLFOX4 or FOLFOX7 with oxaliplatin in a stop-and-Go fashion in advanced colorectal cancer-a GERCOR study. J Clin Oncol. 2006; 24: 394-400.

21.Diaz-Rubio E, Gomez-Espana A, Massuti B, Sastre J, Abad A, Valladares M, et al. First-line XELOX plus bevacizumab followed by XELOX plus bevacizumab or single-agent bevacizumab as maintenance therapy in patients with metastatic colorectal cancer: the phase III MACRO TTD study. Oncologist. 2012; 17: 15-25.

22.Aranda E, Garcia-Alfonso P, Benavides M, Sanchez Ruiz A, Guillen-Ponce C, Safont MJ, et al. First-line mFOLFOX plus cetuximab followed by mFOLFOX plus cetuximab or single-agent cetuximab as maintenance therapy in patients with metastatic colorectal cancer: Phase II randomised MACRO2 TTD study. Eur J Cancer. 2018; 101: 263-72.

23.Simkens LH, van Tinteren H, May A, ten Tije AJ, Creemers GJ, Loosveld OJ, et al. Maintenance treatment with capecitabine and bevacizumab in metastatic colorectal cancer (CAIRO3): a phase 3 randomised controlled trial of the Dutch Colorectal Cancer Group. Lancet. 2015; 385: 1843-52.

24.Luo HY, Li YH, Wang W, Wang ZQ, Yuan X, Ma D, et al. Single-agent capecitabine as maintenance therapy after induction of XELOX (or FOLFOX) in first-line treatment of metastatic colorectal cancer: randomized clinical trial of efficacy and safety. Ann Oncol. 2016; 27: 1074-81. 
25.Klement G, Baruchel S, Rak J, Man S, Clark K, Hicklin DJ, et al. Continuous low-dose therapy with vinblastine and VEGF receptor-2 antibody induces sustained tumor regression without overt toxicity. $J$ Clin Invest. 2000; 105: R15-24.

26.Shi H, Jiang J, Ji J, Shi M, Cai Q, Chen X, et al. Anti-angiogenesis participates in antitumor effects of metronomic capecitabine on colon cancer. Cancer Lett. 2014; 349: 128-35.

27.Kaneno R, Shurin GV, Kaneno FM, Naiditch H, Luo J, Shurin MR. Chemotherapeutic agents in low noncytotoxic concentrations increase immunogenicity of human colon cancer cells. Cell Oncol (Dordr). 2011; 34: 97-106.

28.Kaneno R, Shurin GV, Tourkova IL, Shurin MR. Chemomodulation of human dendritic cell function by antineoplastic agents in low noncytotoxic concentrations. J Transl Med. 2009; 7: 58.

\section{Figures}

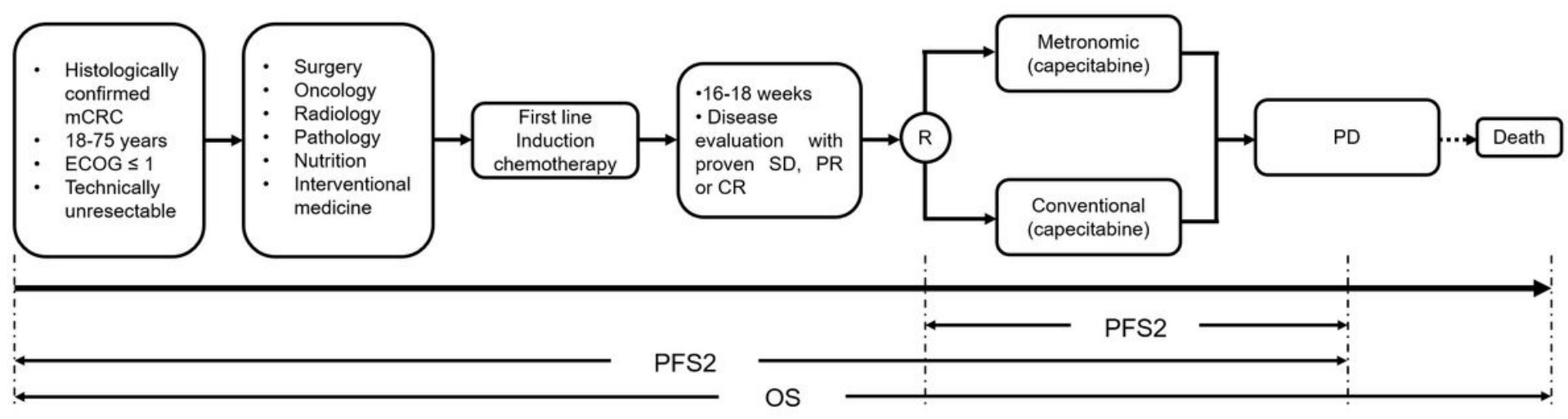

\section{Figure 1}

Study design and flowchart. 


\begin{tabular}{|c|c|c|c|c|c|c|c|c|}
\hline & $\begin{array}{l}\text { First Libe } \\
\text { trentmeat }\end{array}$ & $\begin{array}{l}\text { Middle of } \\
\text { first line } \\
\text { treatmeat }\end{array}$ & $\begin{array}{l}\text { Before } \\
\text { esrollmeat }\end{array}$ & $\begin{array}{r}\text { Der } \\
\text { mainte } \\
\text { treat }\end{array}$ & $\begin{array}{l}\text { ting } \\
\text { enance } \\
\text { tameat }\end{array}$ & & $\begin{array}{c}\text { After } \\
\text { maintenance } \\
\text { treatment }\end{array}$ & \\
\hline & 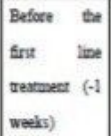 & \begin{tabular}{|l|} 
Ather the frst: \\
line traterest \\
(8-9 wedks)
\end{tabular} & $\begin{array}{l}\text { End of firstine } \\
\text { teatusent (16-18 } \\
\text { areks) }\end{array}$ & $\begin{array}{l}\text { Abe } \\
\text { momecince } \\
\text { reuspent } \\
\text { (every } 4 \text { atels) }\end{array}$ & $\begin{array}{l}\text { Afhe } \\
\text { mainteanse } \\
\text { genmede } \\
\text { (every } 8 \text { weths) }\end{array}$ & $\begin{array}{lr}\text { Whes exiang } \\
\text { the r stady } \\
\text { (within r r } & 1 \\
\text { weeks) } & \end{array}$ & $\begin{array}{l}\text { Abe } \\
\text { withtrowal } \\
\text { foom the snudy } \\
\text { (fourth weeks) }\end{array}$ & Dase of \\
\hline Iaformend cooseat & & & 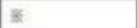 & & & & & \\
\hline $\begin{array}{l}\text { Vital sigas and } \\
\text { physical } \\
\text { eramisation }\end{array}$ & * & 落 & 業 & : & & 等 & 항 & \\
\hline $\begin{array}{l}\text { EORTC QLQ-C30 } \\
\text { Form }\end{array}$ & * & 音 & 亲 & $\mathrm{z}$ & & * & : & \\
\hline Liquid biopsy & 㐘 & : & ; & & 等 & 絭 & 爰 & \\
\hline Routine blood test & 独 & 萃 & 差 & \# & & 紊 & * & \\
\hline $\begin{array}{l}\text { Blood } \\
\text { biocternistry }\end{array}$ & 8 & s: & 萧 & 传 & & 桬 & $\approx$ & \\
\hline
\end{tabular}

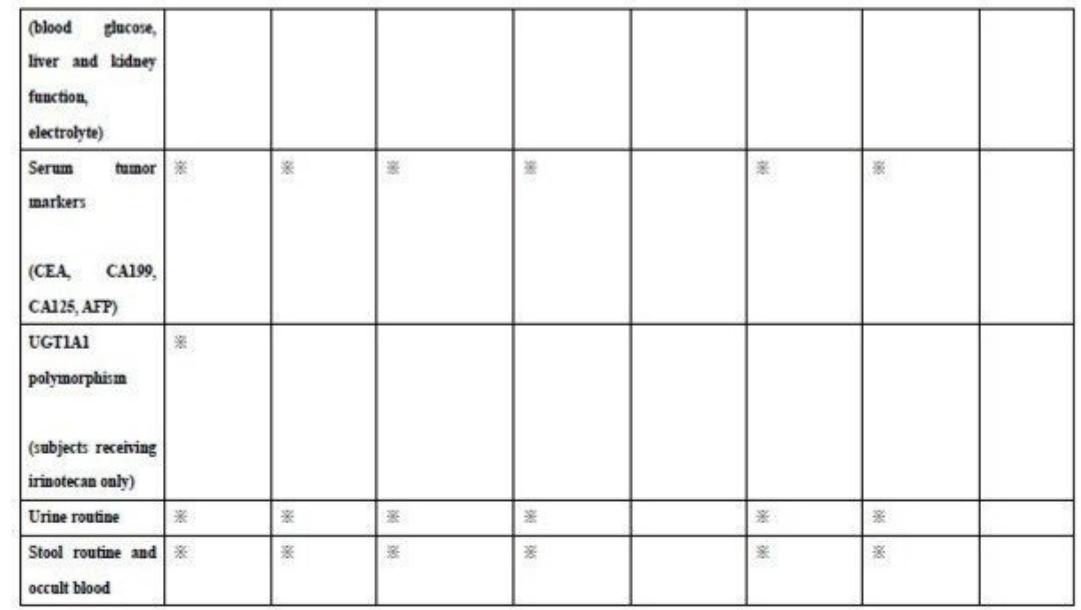

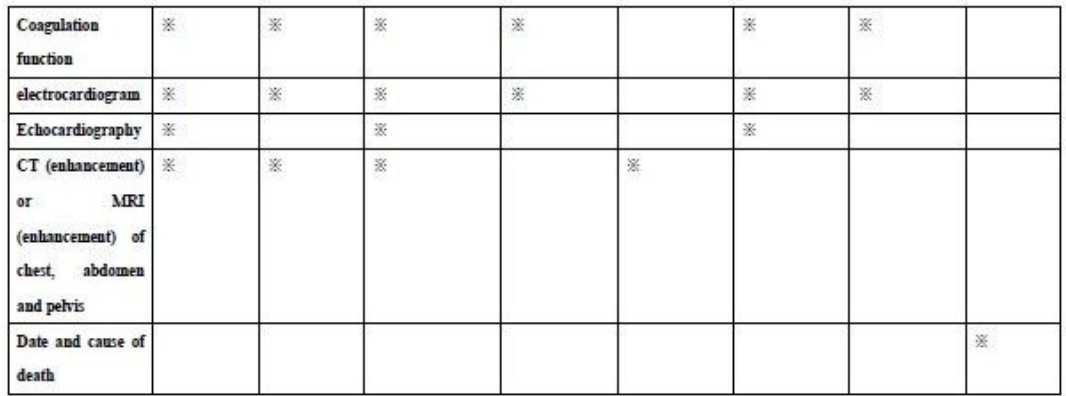

Figure 2

SPIRIT figure

\section{Supplementary Files}

This is a list of supplementary files associated with this preprint. Click to download. 
- SPIRITChecklist.doc

Page 14/14 